\title{
Base pair opening and bubble transport in a DNA double helix induced by a protein molecule in a viscous medium
}

\author{
V. Vasumath:* and M. Danieit \\ Centre for Nonlinear Dynamics, School of Physics, \\ Bharathidasan University, Tiruchirappalli - 620 024, India.
}

(Dated: November 9, 2018)

\begin{abstract}
We study the nonlinear dynamics of a protein-DNA molecular system by treating DNA as a set of two coupled linear chains and protein in the form of a single linear chain sliding along the DNA at the physiological temperature in a viscous medium. The nonlinear dynamics of the above molecular system in general is governed by a perturbed nonlinear Schrödinger equation. In the non-viscous limit, the equation reduces to the completely integrable nonlinear Schrödinger (NLS) equation which admits N-soliton solutions. The soliton excitations of the DNA bases make localized base pair opening and travel along the DNA chain in the form of a bubble. This may represent the bubble generated during the transcription process when an RNA-polymerase binds to a promoter site in the DNA double helical chain. The perturbed NLS equation is solved using a perturbation theory by treating the viscous effect due to surrounding as a weak perturbation and the results show that the viscosity of the solvent in the surrounding damps out the amplitude of the soliton.
\end{abstract}

PACS numbers: 87.15.-v, 05.45.Yv, 02.30.Jr

*Electronic address: vasu@cnld.bdu.ac.in

${ }^{\dagger}$ Electronic address: daniel@cnld.bdu.ac.in 


\section{INTRODUCTION}

Protein-Deoxyribonucleic acid(DNA) interaction plays an important role in a large number of cellular processes such as gene expression, suppression, replication, transcription, recombination, repair and in several other processes [1]. DNA participation in the above processes are mediated or catalyzed by DNA-binding proteins like polymerases, helicases, nucleases, isomerases, ligases and histones. Usually cellular processes start with the binding of a protein (enzyme) to the DNA. For example, the transcription process starts with the binding of RNA-polymerase (an enzyme protein) with a promoter site of the DNA and this binding is known to change the conformation of DNA by opening the bases. The above conformation change or base pair opening stresses the relevance of dynamics in understanding protein-DNA interaction. Experimentally, these conformational changes in DNA during protein-DNA interaction have been studied through atomic force microscopy [2], dynamic spectroscopy [3], X-ray crystallography of protein-DNA co-crystal [4], NMR studies [5] and electrophoresis experiments [6] as well as through fluorescent measurements [7]. Results from free energy calculations combined with molecular dynamics simulations also explain the base flipping through protein-DNA interaction [8, 9, 10, 11]. However, from a theoretical point of view, the understanding of protein-DNA interaction and its dynamics is still in its infancy. This is because the individual dynamics of DNA and protein itself is a very complex one. Also, interaction of the DNA molecule with the surrounding viscous medium and thermal fluctuations add to the complexity. The knowledge of the DNA dynamics through soliton-like excitations describe base pair opening or base flipping during DNA functions. In this direction, Englander et al [12], used soliton excitations to explain base pair opening in DNA for the first time. Yomosa [13, 14] proposed a plane base rotator model by taking into account the rotational motion of bases in a plane normal to the helical axis, and then Takeno and Homma [15, 16] generalized the same and the nonlinear molecular excitations were shown to be governed by kink-antikink solitons. In contrast to the above, Peyrard and Bishop [17] and Christiansen and his collegues [18] proposed a model by taking into account the transverse and longitudinal motions of bases in DNA to describe the base pair opening through breather. Later, several authors [19, 20, 21] including the present authors [22, 23] suggested that either kink soliton or breather would be a good candidate to play a basic role in base pair opening in DNA. In all the above studies, the dynamics of DNA 
molecular system has been studied without taking into account thermal fluctuations and viscosity of the surrounding medium and hence, it was shown that, the soliton representing the base pair opening in DNA may travel for infinite distance and time. Recently, few authors studied the effect of thermal fluctuations [24, 25] and viscosity of the surrounding medium on unzipping and soliton-like base-pair opening and showed that these effects damp the solitons and hence travel only for a limited distance [26, 27, 28, 29, 30]. Like DNA dynamics, the dynamics of protein through soliton excitations also plays an important role in describing energy transfer in alpha helix proteins. In this regard, Davydov [31, 32] who for the first time used soliton excitations to explain energy transfer in alpha helical proteins by proposing a new model by considering the coupling between the quantum transition occurring due to the vibrational structure of the $C=O$ bond and the elastic longitudinal wave propagation along the chain with the helix behaving like a spring. Later on, the above study was extended by several authors [33, 34] including one of the present authors [35, 36] to study the effect of exchange excitation between the chains, temperature, higher order interactions and interspine coupling. In contrast to Davydov's model, Yomosa [37] and very recently Sataric et al [38] studied soliton excitations in protein by considering the molecule as a toda lattice chain. The recent results on the statistical mechanics of protein-DNA system through coarse-grain and worm-like chain models describe unzipping of the DNA chain [39, 40, 41, 42]. Normally, protein molecule interacts with DNA either through non-specific interaction (sequence-independent) which is mainly driven by the electrostatic attractive force between positively charged amino acids and negatively charged phosphate groups of the DNA back bone or through specific interactions (sequence-dependent) including hydrogen bonds, van der Waals force and water mediated bonds between the protein molecule and specific site of DNA [43]. In a recent paper, Sataric et al [44] studied the impact of regulatory proteins through hydrogen bonds on breather excitations in DNA by considering Davydov model of amide-I vibration for protein dynamics and Peyrard-Bishop's model of stretching of hydrogen bonds for DNA dynamics and found that binding of protein to DNA gives rise to a large-amplitude breather. Except the above, no author has so far studied the impact of protein on the dynamics of DNA. Hence, in the present paper, we study the conformational changes that take place in the form of base pair opening in DNA through nonlinear excitations induced by a protein molecule through interaction at the physiological temperature in a viscous surrounding medium. The paper is organized as follows. In section 
II, we present details of the above model and the Hamiltonian for the DNA molecular chain. The model Hamiltonian for protein-DNA interaction is given in section III. In section IV, we derive the equation of motion for the protein-DNA molecular system in the continuum limit and in the next section (section V) base pair opening in DNA as soliton solution of the associated nonlinear dynamical equation in a non-viscous medium is shown. The effect of viscosity of the surrounding medium on the base pair opening in DNA is understood through perturbation analysis in section VI. The results are concluded in section VII.

\section{MODEL HAMILTONIAN FOR DNA DYNAMICS}

We consider a DNA double helix in B-form along with a protein molecule (say RNApolymerase) at physiological temperature in a surrounding viscous medium and study the impact of protein interaction on the dynamics of DNA. The model we propose here for the study treats DNA as a set of two coupled linear molecular chains and protein as a single linear chain interacting through a linear coupling. A schematic representation of the above protein-DNA molecular system is shown in Fig. 1(a). In the figure $R$ and $R^{\prime}$ represent the two complementary strands in the DNA double helix. Each arrow represents the direction of the base attached to the strands and the dots between arrows represent the net hydrogen bonding effect between the complementary bases. The shaded ellipse overlapping the DNA double helical structure represents interaction of protein with the DNA molecule. The conformation and stability of DNA double helix is mainly determined by the stacking of bases through intrastrand dipole-dipole interaction and through hydrogen bonds between the complementary bases (interstrand interaction). From a heuristic argument, it was assumed that the hydrogen bonding energy between the complementary bases depends on the distance between them. Generally, the distance between the complementary bases can be expressed through longitudinal, transverse and rotational motion of bases. Among the different motions, the rotational motion of bases is found to contribute more towards the opening of bases pairs. Hence, for our study, we consider a plane-base rotator model for DNA [13, 15] which the authors have extensively used for studying pure DNA dynamics in the recent times [22, 23]. In order to find the distance between the complementary bases, in Figs. 1(b) and 1(c), horizontal projections of the $n^{\text {th }}$ base pair in the xy and xz-planes are

presented respectively. In the figure, $Q_{n}$ and $Q_{n}^{\prime}$ denote the tip of the $n^{\text {th }}$ bases belonging 


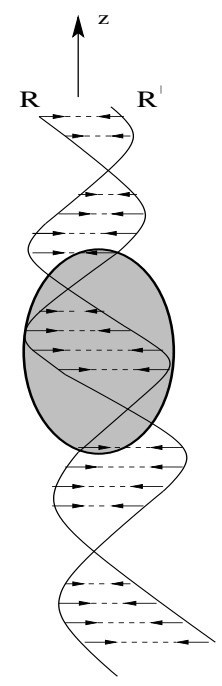

(a)

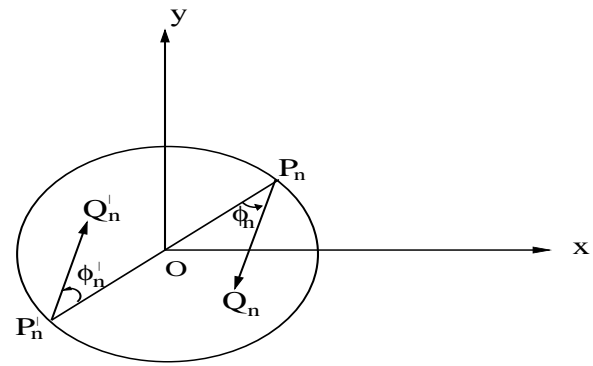

(b)

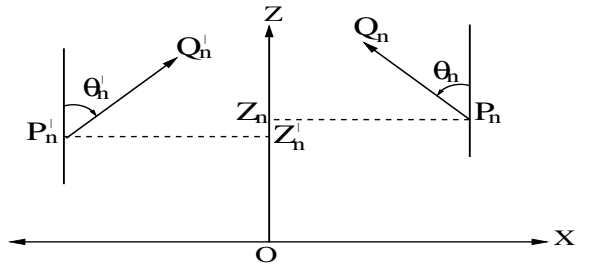

(c)

FIG. 1: (a) A schematic representation of a DNA double helix molecule with a protein molecule interacting with it. (b) A horizontal projection of the $n^{\text {th }}$ base pair of the DNA in the xy-plane. (c) A horizontal projection of the $n^{\text {th }}$ base pair of DNA in the xz-plane.

to the complementary strands $R$ and $R^{\prime}$ at $P_{n}$ and $P_{n}^{\prime}$ respectively. Let $\theta_{n}\left(\theta_{n}^{\prime}\right)$ and $\phi_{n}\left(\phi_{n}^{\prime}\right)$ represent the angles of rotation of the bases in the $n^{\text {th }}$ base pair in the xz and xy-planes respectively. By using simple geometry in Figs. 1(b,c), we can write the distance between the tips of bases as [16]

$$
\begin{aligned}
\left(Q_{n} Q_{n}^{\prime}\right)^{2}= & 2+4 r^{2}+\left(z_{n}-z_{n}^{\prime}\right)^{2}+2\left(z_{n}-z_{n}^{\prime}\right) \\
& \times\left(\cos \theta_{n}-\cos \theta_{n}^{\prime}\right)-4 r\left[\sin \theta_{n} \cos \phi_{n}\right. \\
& \left.+\sin \theta_{n}^{\prime} \cos \phi_{n}^{\prime}\right]+2\left[\operatorname { s i n } \theta _ { n } \operatorname { s i n } \theta _ { n } ^ { \prime } \left(\cos \phi_{n}\right.\right. \\
& \left.\left.\times \cos \phi_{n}^{\prime}+\sin \phi_{n} \sin \phi_{n}^{\prime}\right)-\cos \theta_{n} \cos \theta_{n}^{\prime}\right]
\end{aligned}
$$

where ' $r$ ' is the radius of the circle depicted in Fig. 1(b).

The hydrogen bonding energy can be understood in a more clear and transparent way by introducing quasi-spin operators $\mathbf{S}_{\mathbf{n}} \equiv\left(S_{n}^{x}, S_{n}^{y}, S_{n}^{z}\right)=\left(\sin \theta_{n} \cos \phi_{n}, \sin \theta_{n} \sin \phi_{n}, \cos \theta_{n}\right)$ and $\mathbf{S}_{\mathbf{n}}^{\prime} \equiv\left(S_{n}^{\prime x}, S_{n}^{\prime y}, S_{n}^{\prime z}\right)=\left(\sin \theta_{n}^{\prime} \cos \phi_{n}^{\prime}, \sin \theta_{n}^{\prime} \sin \phi_{n}^{\prime}, \cos \theta_{n}^{\prime}\right)$. Using the above, Eq.(11) can 
be rewritten as

$$
\begin{aligned}
\left(Q_{n} Q_{n}^{\prime}\right)^{2}= & 2+4 r^{2}+2\left[S_{n}^{x} S_{n}^{\prime x}+S_{n}^{y} S_{n}^{\prime y}-S_{n}^{z} S_{n}^{\prime z}\right] \\
& -4 r\left[S_{n}^{x}+S_{n}^{\prime x}\right]
\end{aligned}
$$

While writing Eq.(2), we have chosen $z_{n}=z_{n}^{\prime}$. It is interesting to note that the form of $\left(Q_{n} Q_{n}^{\prime}\right)^{2}$ given in Eq.(2) is the same as the Hamiltonian for a generalized form of the Heisenberg spin model. Therefore, the intrastrand base-base interaction in DNA also can be written using the same consideration. Also, it is reasonable to think that if such a quasi-spin model can be used in this problem, the double strand DNA and the rung-like base pairs can be conceived as a coupled spin chain model or a spin ladder system.

With the above consideration, we are at liberty to use the following Heisenberg model of the Hamiltonian for a coupled spin chain model or spin ladder system with ferromagnetictype exchange interaction between nearest neighbouring spins in the same lattice (equivalent to stacking of bases in one strand i.e. intrastrand interaction) and ferromagnetic or antiferromagnetic rung-coupling (equivalent to hydrogen bonds between complementary bases i.e. interstrand interaction).

$$
H_{D}=-\sum_{n}\left[J\left(\mathbf{S}_{\mathbf{n}} \cdot \mathbf{S}_{\mathbf{n}+\mathbf{1}}+\mathbf{S}_{\mathbf{n}}^{\prime} \cdot \mathbf{S}_{\mathbf{n}+\mathbf{1}}^{\prime}\right)+\mu\left(\mathbf{S}_{\mathbf{n}} \cdot \mathbf{S}_{\mathbf{n}}^{\prime}\right)\right]
$$

Thus, the DNA double helical chain is mapped onto a two coupled spin chain model or a spin ladder system with ferromagnetic legs $(J>0)$ and ferromagnetic $(\mu>0)$ or antiferromagnetic $(\mu<0)$ rungs. Therefore, in Hamiltonian (3) , the terms proportional to $J$ correspond to stacking interaction between the $n^{\text {th }}$ base and its nearest neighbours in the two strands and the last term which is proportional to $\mu$ corresponds to the interstrand interaction or hydrogen bond energy between the complementary bases. In equilibrium, the parameter $\mu$ is expected to be less than zero (corresponding to antiferromagnetic coupling).

As DNA works at the biological temperature, the hydrogen atom attached to the bases are also normally in a thermally excited state. Therefore, it is necessary to generalize the above model into a thermal DNA. Thus, to include the effect of thermal phonons into the system, 
we add the following Hamiltonian.

$$
\begin{aligned}
H_{T} & =\sum_{n}\left[\frac{p_{n}^{2}}{2 m_{1}}+k_{1}\left(X_{n}-X_{n+1}\right)^{2}\right], \\
H_{D-T} & =\alpha_{1} \sum_{n}\left(X_{n+1}-X_{n-1}\right)\left(\mathbf{S}_{\mathbf{n}} \cdot \mathbf{S}_{\mathbf{n}}^{\prime}\right),
\end{aligned}
$$

where $p_{n}=m_{1} \dot{X}_{n}$, with overdot representing the time derivative and $k_{1}$ represents the elastic constant. $m_{1}$ is the mass of the hydrogen atom attached to the base and $X_{n}$ represents the displacement of it at the $n^{\text {th }}$ site along the direction of the hydrogen bond. The interaction Hamiltonian $H_{D-T}$ given in Eq. (4b) represents the coupling between the vibration of the above hydrogen atom (thermal fluctuation) and the rotation of bases.

\section{MODEL HAMILTONIAN FOR PROTEIN-DNA INTERACTION}

As protein binding to DNA induces large mechanical stress on it, which causes conformational changes, and thus acts as a precursor for all the functions of DNA. Hence, we investigate here the dynamics of a DNA when a protein molecule interacts with it by sliding on the DNA chain. Eventhough, proteins are much larger in size than the DNA(substrate), only a small portion of the protein molecule, namely the active site is directly interacting with the DNA molecule (see Fig.1 (a)). Hence, we consider the short active site region of the protein molecule that interacts with the DNA as a linear chain as has been treated by several other authors (see for e.g. Sataric et al [38]). Thus, as said earlier, we propose the model for the above protein-DNA molecular system by considering DNA as a set of two coupled linear chains and the active site region of the protein molecule as a linear molecular chain interacting with the bases which is schematically shown in Fig. 2. The DNA part of the sketch is the linearized chains of Fig. 1(a) and $n, n \pm 1$ represent the $n^{\text {th }}$ and $(n \pm 1)^{t h}$ sites representing the bases (similar to $P_{n}, P_{n}^{\prime}, P_{n \pm 1}, P_{m \pm 1}^{\prime}$ in Figs. 1(b,c)). $G$ represents the linear protein molecular chain (active site region) interacting with the bases by sliding on the DNA chains. To explain the model for the above system further, we consider the protein molecule as a collection of mass points, with each mass point representing a peptide unit and connected by linear springs exhibiting longitudinal stretching motion parallel to the helical axis of DNA, i.e. along z-direction which couples to the hydrogen bonds of bases in a linear way. Hence, the model Hamiltonians for the longitudinal stretching motion of 


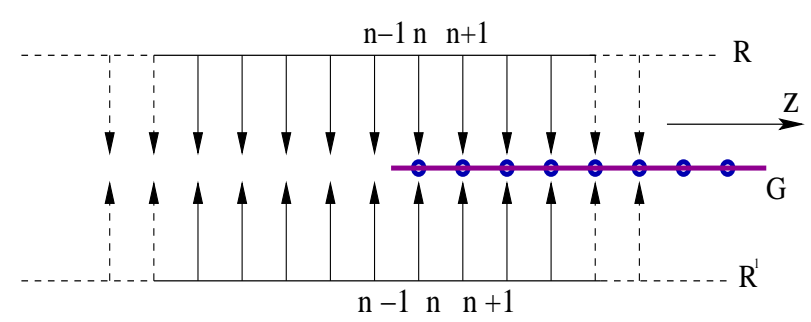

FIG. 2: A sketch representing the protein-DNA molecular system

the protein molecule $\left(H_{p}\right)$ and its interaction with the DNA chain $\left(H_{D-p}\right)$ is written as

$$
\begin{aligned}
H_{p} & =\sum_{n}\left[\frac{q_{n}^{2}}{2 m_{2}}+k_{2}\left(y_{n}-y_{n+1}\right)^{2}\right], \\
H_{D-p} & =\alpha_{2} \sum_{n}\left(y_{n+1}-y_{n-1}\right) S_{n}^{z} S_{n}^{\prime z},
\end{aligned}
$$

where $q_{n}=m_{2} \dot{y}_{n}$, and $m_{2}$ is the mass of the peptide. $y_{n}$ denotes displacement of the $n^{t h}$ peptide in the protein chain from its equilibrium position and $k_{2}$ represents the elastic constant associated with the small amplitude oscillation of the protein molecule. The interaction Hamiltonian $H_{D-p}$ given in Eq.(5b) is chosen to represent the change in hydrogen bonding energy due to change in the distance between the adjacent peptide units along the hydrogen bonding spines of the protein molecule and $\alpha_{2}$ is the coupling coefficient. Further, as the protein molecule is assumed to slide on the DNA chain, the interaction energy along z-direction is expected to be dominant over the energy in the xy-plane normal to it (see Eq.(5b)). Thus, the total Hamiltonian for our model can be written using Eqs. (3i), (4a), (4b), (5a) and (5b) as

$$
\begin{aligned}
H= & H_{D}+H_{p}+H_{D-p}+H_{T}+H_{D-T}, \\
= & \sum_{n}\left[-\left\{J\left(\mathbf{S}_{\mathbf{n}} \cdot \mathbf{S}_{\mathbf{n + 1}}+\mathbf{S}_{\mathbf{n}}^{\prime} \cdot \mathbf{S}_{\mathbf{n}+\mathbf{1}}^{\prime}\right)+\mu\left(\mathbf{S}_{\mathbf{n}} \cdot \mathbf{S}_{\mathbf{n}}^{\prime}\right)\right\}\right. \\
& +\frac{p_{n}^{2}}{2 m_{1}}+\frac{q_{n}^{2}}{2 m_{2}}+k_{1}\left(X_{n}-X_{n+1}\right)^{2}+k_{2}\left(y_{n}-y_{n+1}\right)^{2} \\
& \left.+\alpha_{1}\left(X_{n+1}-X_{n-1}\right)\left(\mathbf{S}_{\mathbf{n}} \cdot \mathbf{S}_{\mathbf{n}}^{\prime}\right)+\alpha_{2}\left(y_{n+1}-y_{n-1}\right) S_{n}^{z} S_{n}^{\prime z}\right] .
\end{aligned}
$$

Before proceeding further, for the sake of completeness, we present the form of the Hamil- 
tonian (6) in terms of angles of rotation of bases as

$$
\begin{aligned}
H= & \sum_{n}\left[-J\left\{\sin \theta_{n} \sin \theta_{n+1} \cos \left(\phi_{n+1}-\phi_{n}\right)+\cos \theta_{n} \cos \theta_{n+1}\right.\right. \\
& \left.+\sin \theta_{n}^{\prime} \sin \theta_{n+1}^{\prime} \cos \left(\phi_{n+1}^{\prime}-\phi_{n}^{\prime}\right)+\cos \theta_{n}^{\prime} \cos \theta_{n+1}^{\prime}\right\} \\
& -\left[\mu-\alpha_{1}\left(X_{n+1}-X_{n-1}\right)\right]\left\{\sin \theta_{n} \sin \theta_{n}^{\prime} \cos \left(\phi_{n}-\phi_{n}^{\prime}\right)+\cos \theta_{n} \cos \theta_{n}^{\prime}\right\} \\
& +\frac{p_{n}^{2}}{2 m_{1}}+\frac{q_{n}^{2}}{2 m_{2}}+k_{1}\left(X_{n}-X_{n+1}\right)^{2}+k_{2}\left(y_{n}-y_{n+1}\right)^{2} \\
& \left.+\alpha_{2}\left(y_{n+1}-y_{n-1}\right) \cos \theta_{n} \cos \theta_{n}^{\prime}\right]
\end{aligned}
$$

In the case of Heisenberg spin systems, when the spin value is large, the spin dynamics is understood either through a classical approach or under semi-classical approximation by bosonizing the Hamiltonian (see for e.g. Ref. [45]). Also, it should be mentioned that creation and annihilation operators were used to represent the Hamiltonian while studying the transport of charge and hole along short DNA molecules [46, 47] and while investigating the nonlinear dynamics of alpha helical protein molecules using the model proposed by Davydov [31]. Therefore, along the same lines, in order to understand the dynamics of the above protein-DNA molecular system, here also, we bosonize the Hamiltonian (66) using Holstein-Primakoff (H-P) representation [48] for quasi spin operators by writing $S_{n}^{+}=\sqrt{2}\left[1-\epsilon^{2} a_{n}^{\dagger} a_{n}\right]^{1 / 2} \epsilon a_{n}, S_{n}^{-}=\sqrt{2} \epsilon a_{n}^{\dagger}\left[1-\epsilon^{2} a_{n}^{\dagger} a_{n}\right]^{1 / 2}, S_{n}^{z}=\left[1-\epsilon^{2} a_{n}^{\dagger} a_{n}\right]$, where $S_{n}^{ \pm}=S_{n}^{x} \pm i S_{n}^{y}$. In the low temperature limit, $a_{n}^{\dagger} a_{n}<<2 S$, and hence, the H-P transformation can be expanded in a power series in terms of the parameter $\epsilon=1 / \sqrt{S}$ as

$$
\begin{aligned}
& S_{n}^{+}=\sqrt{2} \epsilon\left[1-\frac{\epsilon^{2}}{4} a_{n}^{\dagger} a_{n}-\frac{\epsilon^{4}}{32} a_{n}^{\dagger} a_{n} a_{n}^{\dagger} a_{n}-O\left(\epsilon^{6}\right)\right] a_{n}, \\
& S_{n}^{-}=\sqrt{2} \epsilon a_{n}^{\dagger}\left[1-\frac{\epsilon^{2}}{4} a_{n}^{\dagger} a_{n}-\frac{\epsilon^{4}}{32} a_{n}^{\dagger} a_{n} a_{n}^{\dagger} a_{n}-O\left(\epsilon^{6}\right)\right],
\end{aligned}
$$

and similar expansions for $S_{n}^{\prime+}, S_{n}^{\prime-}$ and $S_{n}^{\prime z}$ in terms of $b_{n}\left(b_{n}^{\dagger}\right)$. Here $a_{n}^{\dagger}\left(b_{n}^{\dagger}\right)$ and $a_{n}\left(b_{n}\right)$ represent creation and annihilation operators of the $n^{\text {th }}$ bases and satisfy the usual commutation relations, $\left[a_{m}, a_{n}^{\dagger}\right]=\left[b_{m}, b_{n}^{\dagger}\right]=\delta_{m n},\left[a_{m}, a_{n}\right]=\left[b_{m}, b_{n}\right]=\left[a_{m}^{\dagger}, a_{n}^{\dagger}\right]=\left[b_{m}^{\dagger}, b_{n}^{\dagger}\right]=0$. Substituting 


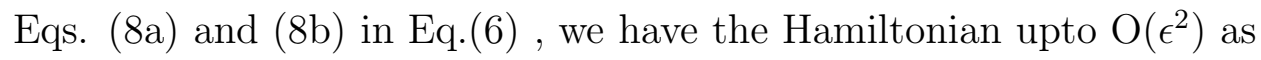

$$
\begin{aligned}
H= & \sum_{n}\left[\frac{p_{n}^{2}}{2 m_{1}}+\frac{q_{n}^{2}}{2 m_{2}}+k_{1}\left(X_{n}-X_{n+1}\right)^{2}+k_{2}\left(y_{n}-y_{n+1}\right)^{2}\right. \\
& +\epsilon^{2}\left\{-J\left(a_{n} a_{n+1}^{\dagger}+a_{n}^{\dagger} a_{n+1}-a_{n}^{\dagger} a_{n}-a_{n+1}^{\dagger} a_{n+1}\right.\right. \\
& \left.+b_{n} b_{n+1}^{\dagger}+b_{n}^{\dagger} b_{n+1}-b_{n}^{\dagger} b_{n}-b_{n+1}^{\dagger} b_{n+1}\right) \\
& -\left[\mu-\alpha_{1}\left(X_{n+1}-X_{n-1}\right)\right]\left(a_{n} b_{n}^{\dagger}+a_{n}^{\dagger} b_{n}-a_{n}^{\dagger} a_{n}\right. \\
& \left.\left.\left.-b_{n}^{\dagger} b_{n}\right)-\alpha_{2}\left(y_{n+1}-y_{n-1}\right)\left(a_{n}^{\dagger} a_{n}+b_{n}^{\dagger} b_{n}\right)\right\}\right] .
\end{aligned}
$$

\section{THE DYNAMICAL EQUATIONS}

Having written down the Hamiltonian in the semi-classical description, the dynamics

of the protein-DNA molecular system can be understood by constructing the equations of motion as

$$
\begin{aligned}
i \hbar \frac{\partial a_{n}}{\partial t} & =\left[a_{n}, H\right] \\
& =F\left(a_{n}^{\dagger}, a_{n}, a_{n+1}^{\dagger}, a_{n+1}\right)
\end{aligned}
$$

and similar one for $b_{n}$. The equations of motion for $X_{n}$ and $y_{n}$ are written using the Hamilton's equations of motion $\frac{\partial X_{n}}{\partial t}=-\frac{\partial H}{\partial p_{n}}, \frac{\partial p_{n}}{\partial t}=\frac{\partial H}{\partial X_{n}}, \frac{\partial y_{n}}{\partial t}=-\frac{\partial H}{\partial q_{n}}$ and $\frac{\partial q_{n}}{\partial t}=\frac{\partial H}{\partial y_{n}}$. The explicit form of the equations of motion can be derived by substituting Hamiltonian (9) in the above equations of motion for $a_{n}, b_{n}, X_{n}$ and $y_{n}$. Thus, we get

$$
\begin{aligned}
i \frac{\partial a_{n}}{\partial t}= & -J\left(a_{n+1}-2 a_{n}+a_{n-1}\right)-\left[\mu+\alpha_{1}\left(X_{n+1}\right.\right. \\
& \left.\left.-X_{n-1}\right)\right]\left(b_{n}-a_{n}\right)-\alpha_{2}\left(y_{n+1}-y_{n-1}\right) a_{n}, \\
i \frac{\partial b_{n}}{\partial t}= & -J\left(b_{n+1}-2 b_{n}+b_{n-1}\right)-\left[\mu+\alpha_{1}\left(X_{n+1}\right.\right. \\
& \left.\left.-X_{n-1}\right)\right]\left(a_{n}-b_{n}\right)-\alpha_{2}\left(y_{n+1}-y_{n-1}\right) b_{n}, \\
m_{1} \frac{\partial^{2} X_{n}}{\partial t^{2}}= & k_{1}\left(X_{n+1}-2 X_{n}+X_{n-1}\right)+\alpha_{1}\left[a_{n-1}^{\dagger} a_{n-1}\right. \\
& -a_{n+1}^{\dagger} a_{n+1}+b_{n-1}^{\dagger} b_{n-1}-b_{n+1}^{\dagger} b_{n+1} \\
& \left.a_{n+1} b_{n+1}^{\dagger}-a_{n-1} b_{n-1}^{\dagger}+a_{n+1}^{\dagger} b_{n+1}-a_{n-1}^{\dagger} b_{n-1}\right], \\
m_{2} \frac{\partial^{2} y_{n}}{\partial t^{2}}= & k_{2}\left(y_{n+1}-2 y_{n}+y_{n-1}\right)+\alpha_{2}\left[a_{n-1}^{\dagger} a_{n-1}\right. \\
& \left.-a_{n+1}^{\dagger} a_{n+1}+b_{n-1}^{\dagger} b_{n-1}-b_{n+1}^{\dagger} b_{n+1}\right] .
\end{aligned}
$$


While writing the above equations $(11 \mathrm{a}, 11 \mathrm{~d})$, we have rescaled the time variable and redefined $m_{1}, m_{2}, k_{1}$ and $k_{2}$. In order to represent the large amplitude collective modes by coherent states, we introduce Glauber's coherent state representation [49] for boson operators $a_{n}^{\dagger}\left|u>=u_{n}^{*}\right| u>, a_{n}\left|u>=u_{n}\right| u>,\left|u>=\Pi_{n}\right| u_{n}>$ and $b_{n}^{\dagger}\left|v>=v_{n}^{*}\right| v>, b_{n}\left|v>=v_{n}\right| v>$ ,$|v\rangle=\Pi_{n}\left|v_{n}\right\rangle$ with $\langle u \mid u\rangle=1$ and $\langle v \mid v\rangle=1$ where $u_{n}$ and $v_{n}$ are the coherent amplitudes of the operators $a_{n}$ and $b_{n}$ for the system in the states $\mid u>$ and $\mid v>$ respectively. Further, as the length of the DNA and the protein chains are very large compared to the lattice parameter, we make a continuum approximation by introducing the new fields $u_{n} \rightarrow u(z, t), v_{n}(t) \rightarrow v(z, t), X_{n}(t) \rightarrow X(z, t)$ and $y_{n}(t) \rightarrow y(z, t)$, where $z=n l$ with the expansions $u_{n \pm 1}=u(z, t) \pm l \frac{\partial u}{\partial z}+\frac{l^{2}}{2 !} \frac{\partial^{2} u}{\partial z^{2}} \pm O\left(l^{3}\right)$ and similar ones for $v_{n \pm 1}, X_{n \pm 1}$ and $y_{n \pm 1}$. Under the above approximations, the equations of motion (11a), (11b), (11c) and (11d) after rescaling $z$ and redefining $\alpha_{1}, \alpha_{2}, k_{1}$ and $k_{2}$ upto $O\left(l^{2}\right)$ can be written as

$$
\begin{aligned}
i u_{t} & =-u_{z z}-\left(\mu-\alpha_{1} X_{z}\right)(v-u)-\alpha_{2} y_{z} u \\
i v_{t} & =-v_{z z}-\left(\mu-\alpha_{1} X_{z}\right)(u-v)-\alpha_{2} y_{z} v \\
m_{1} X_{t t} & =k_{1} X_{z z}-\alpha_{1}\left[|u|^{2}+|v|^{2}-u v^{*}-u^{*} v\right]_{z} \\
m_{2} y_{t t} & =k_{2} y_{z z}-\alpha_{2}\left[|u|^{2}+|v|^{2}\right]_{z} .
\end{aligned}
$$

In Eqs. (12a-12d), the suffices $t$ and $z$ represent partial derivatives with respect to time $t$ and the spatial variable $z$ respectively. On subtracting Eq. (12a) from (12b) and by choosing $v=-u$, Eqs. 12 (a-d) can be written as

$$
\begin{array}{r}
i u_{t}-\left\{2 \mu-\left(\alpha_{2} y_{z}-2 \alpha_{1} X_{z}\right)\right\} u+u_{z z}=0, \\
X_{t t}-\frac{k_{1}}{m_{1}} X_{z z}=-\frac{4 \alpha_{1}}{m_{1}}\left[|u|^{2}\right]_{z}, \\
y_{t t}-\frac{k_{2}}{m_{2}} y_{z z}=-\frac{2 \alpha_{2}}{m_{2}}\left[|u|^{2}\right]_{z} .
\end{array}
$$

It may be mentioned that, addition of Eqs. (12a) and (12b) satisfy identically. The term proportional to $\mu$ in Eq. (13a) can be transformed away using the transformation $u(z, t)=$ $\hat{u}(z, t) e^{-2 i \mu t}$ and the result reads (after dropping the hat)

$$
i u_{t}+u_{z z}+\left(2 \alpha_{1} X_{z}+\alpha_{2} y_{z}\right) u=0
$$

The set of coupled equations (13b,c) and (14) describe the dynamics of our protein-DNA molecular system at the biological temperature, when the protein molecule binds to the 
DNA double helical chain through linear harmonic coupling. The dynamics is found to be governed by the excitation of DNA bases and thermal vibration of the hydrogen atoms attached to the bases combined with the longitudinal motion of peptide units of the binding protein. In particular, we are concerned with the nonlinear excitation of bases induced by protein and thermal fluctuations, in which a cluster of DNA bases may undergo a large excursion as compared to the rest of the bases. It may be noted that when $\alpha_{1}=\alpha_{2}=0$, Eqs. (14), (13b) and (13c) are decoupled and reduced to a set of linear equations. Thus, when the protein molecule is detached from the DNA chain $\left(\alpha_{1}=\alpha_{2}=0\right)$, the dynamics is governed by the following well known set of linear equations.

$$
\begin{aligned}
i u_{t}+u_{z z} & =0, \\
X_{t t}-\frac{k_{1}}{m_{1}} X_{z z}=0, y_{t t}-\frac{k_{2}}{m_{2}} y_{z z} & =0 .
\end{aligned}
$$

While Eq. (15a) is the time-dependent Schrödinger equation for a free particle, Eqs.(15b) are the homogeneous linear wave equations. Eq. (15a) admits plane transverse wave solution of the form $u=u_{0} e^{i(\kappa z-w t)}$ with the dispersion relation $w=\kappa^{2}$ where $u_{0}$ is the constant amplitude. On the other hand, Eq. (15b) admits linear non-dispersive wave solutions $X=f_{1}\left(z-v_{1} t\right)+g_{1}\left(z+v_{1} t\right)$ and $y=f_{2}\left(z-v_{2} t\right)+g_{2}\left(z+v_{2} t\right)$ where $f_{1}, g_{1}$ and $f_{2}, g_{2}$ are

arbitrary functions and $v_{1}=\sqrt{\frac{k_{1}}{m_{1}}}$ and $v_{2}=\sqrt{\frac{k_{2}}{m_{2}}}$, represent the constant phase velocities of the wave. When the protein molecule started interacting with the DNA molecular chain at the physiological temperature, i.e when $\alpha_{1} \neq 0$ and $\alpha_{2} \neq 0$, the excitation energy of the protein-DNA molecular system at the physiological temperature (thermal fluctuation) increases, and nonlinearity started playing its role. Thus, the set of full coupled nonlinear equations become important and it is essential that Eqs. (13b, c) and (14) should be solved in their full form to understand the underlying nonlinear dynamics.

\section{SOLITON, BASE PAIR OPENING AND BUBBLE TRANSPORT}

In order to solve the set of coupled equations (14), (13b) and (13c), in their full form we differentiate Eqs. (13b) and (13c) with respect to $z$ once and define $\hat{X}(z, t)=X_{z}$ and 
$Y(z, t)=y_{z}$, so that Eqs. (14), (13b) and (13c) are written as

$$
\begin{aligned}
i u_{t}+u_{z z}+\left(2 \alpha_{1} \hat{X}+\alpha_{2} Y\right) u & =0 \\
\hat{X}_{t t}-v_{1}^{2} \hat{X}_{z z}+\frac{4 \alpha_{1}}{m_{1}}\left[|u|^{2}\right]_{z z} & =0 \\
Y_{t t}-v_{2}^{2} Y_{z z}+\frac{2 \alpha_{2}}{m_{2}}\left[|u|^{2}\right]_{z z} & =0 .
\end{aligned}
$$

Now, we rewrite Eqs. (16b) and (16c) by introducing the wave variable $\zeta=z-v_{3} t$ and writing $\hat{X}(z, t) \rightarrow \hat{X}(\zeta), Y(z, t) \rightarrow Y(\zeta)$.

$$
\begin{aligned}
\hat{X}_{\zeta \zeta}-2 \beta_{1}\left[|u|^{2}\right]_{\zeta \zeta} & =0, \\
y_{\zeta \zeta}-2 \beta_{2}\left[|u|^{2}\right]_{\zeta \zeta} & =0
\end{aligned}
$$

where $\beta_{1}=\frac{2 \alpha_{1}}{m_{1}\left(v_{1}^{2}-v_{3}^{2}\right)}$ and $\beta_{2}=\frac{\alpha_{2}}{m_{2}\left(v_{2}^{2}-v_{3}^{2}\right)}$. On integrating Eqs. (17a) and (17b) with respect to $\zeta$ twice and assuming both the integration constants to be zero, we get $\hat{X}=2 \beta_{1}|u|^{2}$ and $Y=2 \beta_{2}|u|^{2}$, which upon using in Eq. (16a) gives

$$
i U_{t}+U_{z z}+2|U|^{2} U=0
$$

While writing Eq. (18), we have made use of the transformation $u(z, t)=\left(2 \alpha_{1} \beta_{1}+\right.$ $\left.\alpha_{2} \beta_{2}\right)^{-\frac{1}{2}} U(z, t)$. Eq. (18) is the well known completely integrable nonlinear Schrödinger (NLS) equation which has been solved for N-soliton solutions using the inverse scattering transform (IST) method [50]. For instance, the one soliton solution is written as

$$
U=\eta \operatorname{sech}\left[\eta\left(z-2 \xi t-\theta_{0}\right)\right] \exp \left[i \xi\left(z-2 \xi t-\theta_{0}\right)+i\left(\left(\eta^{2}+\xi^{2}\right) t-\sigma_{0}\right)\right]
$$

where $\eta, \xi, \theta_{0}$ and $\sigma_{0}$ are four real parameters which determine the propagating amplitude, velocity, initial position and initial phase of the soliton. The solitons in the protein-DNA molecular system with thermal fluctuation are formed as a result of the dynamical balance between the dispersion due to interaction of intrastrand (stacking) dipole vibrations in each strand of the DNA with the nonlinearity provided by the interaction between the hydrogen bonds in DNA and the local displacement of the peptide groups in the protein molecule and the thermal phonons. The longitudinal waves that arise in the protein molecule and hydrogen atoms in DNA in turn provide a potential well that prevents dispersion of the rotational energy of the bases in DNA. Thus, the propagation of rotation of bases in DNA is coupled to the longitudinal waves of protein and the coupled excitations propagate as a 

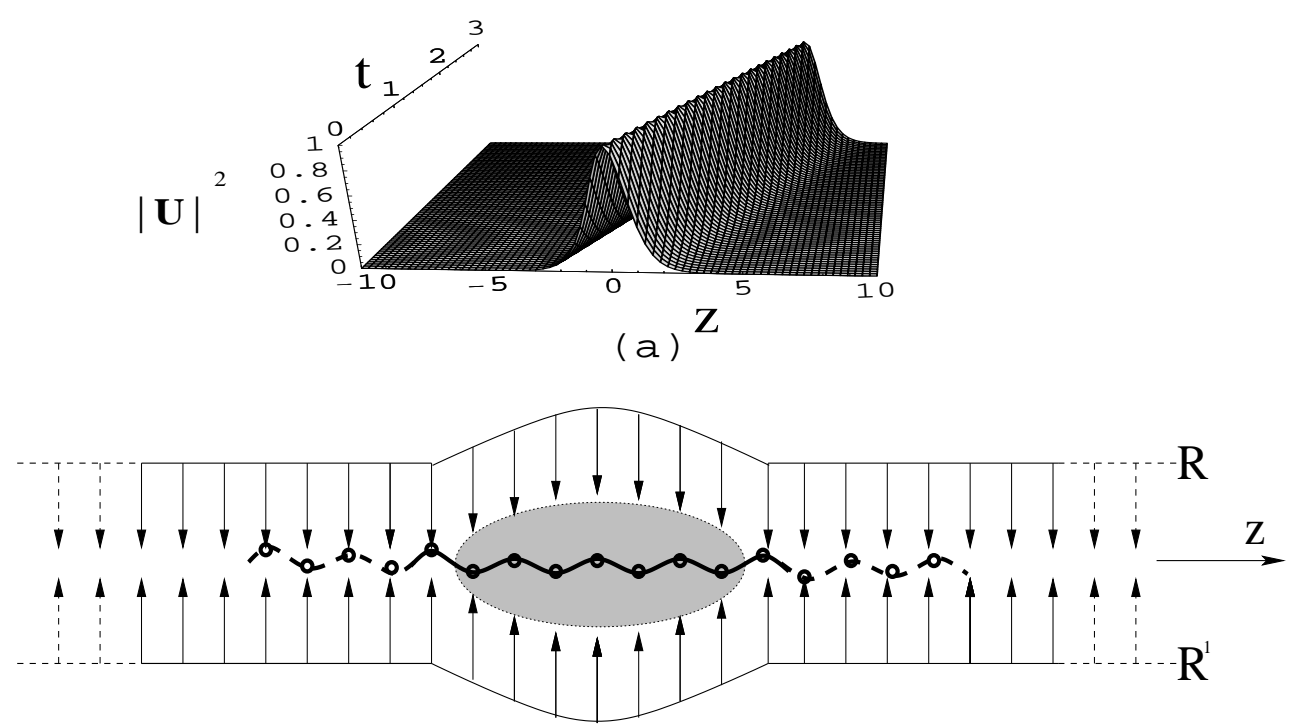

(b)

FIG. 3: (a) One soliton solution ( Eq. 19) of the NLS equation . (b) A schematic representation of formation of bubble with the solitons and its propagation along DNA.

localized and dynamically self-sufficient entity called solitons which travel along each strand of the DNA chain. In Fig. 3(a), we have plotted the square of the absolute value of the one soliton solution $U$ i.e. $|U|^{2}$ as given in Eq. (19). In Fig. 3(b), we present a schematic representation of the coherent base excitations in DNA in terms of rotation of bases induced by the protein molecule in the form of solitons propagating along the two strands which collectively form a travelling bubble created by energy delocalization due to nonlinear effects. Thus, the soliton solution describes an open state configuration in the individual strands of the DNA double helix which collectively represent a bubble. In the figure, the shaded ellipse represents the region of interaction of the protein molecule with DNA where the bubble is formed. Thus, the protein molecule acts as a zip-runner in opening the bases in DNA chain during the process of transcription. Similar results have been observed experimentally by $\mathrm{Ha}$ et al[51], on the winding and unwinding of E-coli Rep helicase-DNA complex. Further, our results on bubble propagation in DNA due to protein interaction is in accordance with the experimental data on the binding of RNApolymerase to promoter [52, 53, 54, 55, 56]. From the expression for the soliton namely $u=\left(2 \alpha_{1} \beta_{1}+\alpha_{2} \beta_{2}\right)^{-\frac{1}{2}} \eta \operatorname{sech}\left[\eta\left(z-2 \xi t-\theta_{0}\right)\right] \exp \left[i \xi\left(z-2 \xi t-\theta_{0}\right)+i\left(\left(\eta^{2}+\xi^{2}\right) t-\sigma_{0}\right)\right]$, it is noted that the amplitude of the soliton depends on the coupling of the DNA excitations to 
the thermal phonons $\left(\alpha_{1}\right)$ and to the molecular vibrations of the protein $\left(\alpha_{2}\right)$ which were also respossible for nonlinearity in the soliton equation. For large coupling, it is expected that the amplitude of the soliton decreases. Recently Campa [24] also showed through simulation studies that, in the case of large thermal coupling, the bubble travels only for a short distance with decreasing amplitude.

\section{EFFECT OF VISCOSITY}

In a more realistic description of the dynamics of protein-DNA system, it is important to consider the effect of the surrounding medium or environment. Effectively, the interaction of DNA with the surrounding medium reduces to viscous damping effect. It is known that, in the case of a protein-DNA system the solvating water acts as a viscous medium that makes the nucleotide oscillations to damp out [57]. The effect of viscous force exerted on the DNA chain can be taken into account by adding a term of the form $-i \gamma U$ to the right-hand side of the completely integrable nonlinear Schrödinger equation (19) which now becomes a damped nonlinear Schrödinger equation as given below.

$$
i U_{t}+U_{z z}+2|U|^{2} U=-i \gamma U
$$

As the viscosity of water is temperature dependent, from a simple fluid mechanics argument, one can estimate the magnitude of the damping coefficient as very small at the physiological temperature [28]. Hence, we treat the term proportional to $\gamma$ in Eq. (20) as a weak perturbation. When $\gamma=0 \mathrm{Eq}$. (20) reduces to the completely integrable NLS equation as given in Eq. (18) and the one soliton solution is given in Eq. (19) which can be rewritten for convenience in the form

$$
U=\eta \operatorname{sech} \eta\left(\theta-\theta_{0}\right) \exp \left[i \xi\left(\theta-\theta_{0}\right)+i\left(\sigma-\sigma_{0}\right)\right]
$$

where $\frac{\partial \theta}{\partial t}=-2 \xi, \frac{\partial \theta}{\partial x}=1, \frac{\partial \sigma}{\partial t}=\eta^{2}+\xi^{2}$ and $\frac{\partial \theta}{\partial x}=0$.

We carry out a perturbation analysis [58] to understand the impact of the viscous force by introducing a slow time variable $T=\gamma t$ and treat the quantities $\eta, \xi, \theta_{0}$ and $\sigma_{0}$ as functions of this time scale and hence the envelope soliton solution (21) is written as

$$
U=\hat{U}(\theta, T ; \gamma) \exp \left[i \xi\left(\theta-\theta_{0}\right)+i\left(\sigma-\sigma_{0}\right)\right]
$$


Under the above assumption of quasi-stationarity, Eq. (20) reads

$$
\eta^{2} \hat{U}+\hat{U}_{\theta \theta}+2|\hat{U}|^{2} \hat{U}=\gamma F(\hat{U})
$$

where

$$
F(\hat{U})=\left[\left(\theta-\theta_{0}\right) \xi_{T}-\xi \theta_{0 T}-\sigma_{0 T}\right] \hat{U}-i\left[\hat{U}_{T}+\hat{U}\right]
$$

We assume Poincaré-type asymptotic expansion for $\hat{U}$ as $\hat{U}(\theta, T ; \gamma)=\sum_{n=1}^{\infty} \gamma^{n} \hat{U}_{n}(\theta, T)$ and further restrict ourselves to calculation of order $(\gamma)$ such that $\hat{U}(\theta, T ; \gamma)=\hat{U}_{0}(\theta, T)+$ $\gamma \hat{U}_{1}(\theta, T)$, where $\hat{U}_{0}=\eta \operatorname{sech}\left[\eta\left(\theta-\theta_{0}\right)\right]$. Further, we assume that $\hat{U}_{1}=\phi_{1}+i \psi_{1}$, where $\phi_{1}$ and $\psi_{1}$ are real. On substituting the above, in Eqs. (23) and (24), we obtain

$$
\begin{aligned}
& L_{1} \phi_{1} \equiv-\eta^{2} \phi_{1}+\phi_{1 \theta \theta}+6\left|\hat{U_{0}}\right|^{2} \phi_{1}=\operatorname{Re} F\left(\hat{U}_{0}\right), \\
& L_{2} \psi_{1} \equiv-\eta^{2} \psi_{1}+\psi_{1 \theta \theta}+2\left|U_{0}\right|^{2} \psi_{1}=\operatorname{Im} F\left(\hat{U}_{0}\right),
\end{aligned}
$$

where

$$
\begin{aligned}
& \operatorname{Re} F\left(\hat{U}_{0}\right)=\left[\left(\theta-\theta_{0}\right) \xi_{T}-\xi \theta_{0 T}-\sigma_{0 T}\right] \hat{U}_{0} \\
& \operatorname{Im} F\left(\hat{U}_{0}\right)=-\left[\hat{U}_{0 T}+\hat{U}_{0}\right] .
\end{aligned}
$$

In Eqs. (25a $)$ and (25b), $L_{1}$ and $L_{2}$ are self-adjoint operators. It may be checked that, the solutions of the homogeneous part of Eqs. (25a and (25b) are $\hat{U}_{0 \theta}$ and $\hat{U}_{0}$ and hence we have the following secularity conditions.

$$
\begin{gathered}
\int_{-\infty}^{\infty} \hat{U}_{0 \theta} \operatorname{Re} F\left(\hat{U}_{0}\right) d \theta=0 \\
\int_{-\infty}^{\infty} \hat{U}_{0} \operatorname{Im} F\left(\hat{U}_{0}\right) d \theta=0 .
\end{gathered}
$$

On evaluating the above integrals after substituting the values of $\hat{U}_{0 \theta}, \hat{U}_{0}, \operatorname{Re} F\left(\hat{U}_{0}\right)$ and $\operatorname{Im} F\left(\hat{U}_{0}\right)$, we obtain $\xi_{T}=0, \eta_{T}=-2 \eta$, which can be written in terms of the original time variable $t$ after integrating once as

$$
\xi=\xi_{0}, \eta=\eta_{0} e^{-2 \gamma t}
$$

where $\eta_{0}$ and $\xi_{0}$ are the initial amplitude and velocity of the soliton. The first of Eq. (28) says that when the protein-DNA system interacts with the surrounding viscous medium the velocity of the soliton remains constant. However, the second of Eq. (28) says that, the 
amplitude of the soliton decreases as soliton propagates. In other words, the viscous nature of the solvent medium damps out the soliton exponentially and hence the soliton is expected to travel only a short distance.

Now, we construct the perturbed soliton by solving Eqs. (25a) and (25b) . For that, first we solve the homogeneous part of Eq. (25a), which admits the following two particular solutions.

$$
\begin{aligned}
\phi_{11}= & \operatorname{sech} \eta\left(\theta-\theta_{0}\right) \tanh \eta\left(\theta-\theta_{0}\right), \\
\phi_{12}= & \frac{1}{\eta}\left[\frac{3}{2} \eta\left(\theta-\theta_{0}\right) \operatorname{sech} \eta\left(\theta-\theta_{0}\right) \tanh \eta\left(\theta-\theta_{0}\right)\right. \\
& +\frac{1}{2} \tanh \eta\left(\theta-\theta_{0}\right) \sinh \eta\left(\theta-\theta_{0}\right) \\
& \left.-\operatorname{sech} \eta\left(\theta-\theta_{0}\right)\right] .
\end{aligned}
$$

The general solution can be found out by using the following expression.

$$
\begin{aligned}
\phi_{1}= & C_{1} \phi_{11}+C_{2} \phi_{12}-\phi_{11} \int_{-\infty}^{\theta} \phi_{12} \operatorname{Re} F\left(\hat{U}_{0}\right) d \theta \\
& +\phi_{12} \int_{-\infty}^{\theta} \phi_{11} \operatorname{Re} F\left(\hat{U}_{0}\right) d \theta
\end{aligned}
$$

Here $C_{1}$ and $C_{2}$ are arbitrary constants. We construct $\phi_{1}$ by substituting the values of $\phi_{11}, \phi_{12}$ and $\operatorname{Re} F\left(\hat{U}_{0}\right)$ given in Eqs. (29a), (29b) and (26a) in Eq. (30) and after evaluating the integrals, we get

$$
\begin{aligned}
\phi_{1}= & -\frac{1}{\eta}\left[C_{2}+\frac{1}{2}\left(\xi \theta_{0 T}+\sigma_{0 T}\right)\right] \operatorname{sech} \eta\left(\theta-\theta_{0}\right)+\left[C_{1}+\frac{3 C_{2}}{2}\left(\theta-\theta_{0}\right)+\frac{1}{2}\left(\theta-\theta_{0}\right)\right. \\
& \left.\left(\xi \theta_{0 T}+\sigma_{0 T}\right)\right] \operatorname{sech} \eta\left(\theta-\theta_{0}\right) \tanh \eta\left(\theta-\theta_{0}\right)+\frac{C_{2}}{2 \eta} \sinh \eta\left(\theta-\theta_{0}\right) \tanh \eta\left(\theta-\theta_{0}\right) .
\end{aligned}
$$

The last term in Eq.(31) is a secular term that leads to a solution which is unbounded and hence, it is removed by choosing the arbitrary constant $C_{2}=0$. Further, by applying the boundary conditions $\left.\phi_{1}\right|_{\theta=\theta_{0}}=$ constant $=c$ and $\left.\phi_{1 \theta}\right|_{\theta=\theta_{0}}=0$, we obtain $\frac{1}{\eta}\left(\xi \theta_{0 T}+\sigma_{0 T}\right)=-c$ and $C_{1}=0$. Using the above results in Eq. (31), the general solution $\phi_{1}$ is written as

$$
\phi_{1}=c\left[1-\left(\theta-\theta_{0}\right) \tanh \eta\left(\theta-\theta_{0}\right)\right] \operatorname{sech} \eta\left(\theta-\theta_{0}\right) \text {. }
$$

Next, we solve Eq.(25b), the homogeneous part of which admits the following particular solutions.

$$
\begin{aligned}
& \psi_{11}=\operatorname{sech} \eta\left(\theta-\theta_{0}\right) \\
& \psi_{12}=\frac{1}{2 \eta}\left[\eta\left(\theta-\theta_{0}\right) \operatorname{sech} \eta\left(\theta-\theta_{0}\right)+\sinh \eta\left(\theta-\theta_{0}\right)\right]
\end{aligned}
$$


Knowing two particular solutions, the general solution of Eq.(25b) can be found from

$$
\begin{aligned}
\psi_{1}= & C_{3} \psi_{11}+C_{4} \psi_{12}-\psi_{11} \int_{-\infty}^{\theta} \psi_{12} \operatorname{Im} F\left(\hat{U}_{0}\right) d \theta \\
& +\psi_{12} \int_{-\infty}^{\theta} \psi_{11} \operatorname{Im} F\left(\hat{U}_{0}\right) d \theta,
\end{aligned}
$$

where $C_{3}$ and $C_{4}$ are arbitrary constants. We construct the explicit form of $\psi_{1}$ by substituting the values of $\psi_{11}, \psi_{12}$ and $\operatorname{Im} F\left(\hat{U}_{0}\right)$ given in Eqs. (33a), (33b) and (26b) and evaluating the integrals.

$$
\begin{aligned}
\psi_{1}= & \left\{C_{3}+\frac{C_{4}}{2}\left(\theta-\theta_{0}\right)-\frac{\eta}{2}\left[\left(\theta-\theta_{0}\right)\left\{\frac{\eta_{T}}{2 \eta}\left(\theta-\theta_{0}\right)-\theta_{0 T}\right\}+\theta_{0 T} \tanh \eta\left(\theta-\theta_{0}\right)\right.\right. \\
& \left.\left.+\theta_{0 T}\left(\theta-\theta_{0}\right) \operatorname{sech}^{2} \eta\left(\theta-\theta_{0}\right)\right]\right\} \operatorname{sech}\left(\theta-\theta_{0}\right) \\
& +\frac{C_{4}}{2 \eta} \sinh \eta\left(\theta-\theta_{0}\right) .
\end{aligned}
$$

The above solution for $\psi_{1}$ contains secular term that is a term proportional to $\sinh \eta\left(\theta-\theta_{0}\right)$ which can be removed by choosing $C_{4}=0$. Further, we obtain $C_{3}=0$ and $\theta_{0 T}=0$ upon using the boundary conditions $\left.\psi_{1}\right|_{\theta=\theta_{0}}=0$ and $\left.\psi_{1 \theta}\right|_{\theta=\theta_{0}}=0$. On using the above results in Eq. (35), the final form of $\psi_{1}$ is written as

$$
\psi_{1}=\frac{\eta}{2}\left(\theta-\theta_{0}\right)^{2} \operatorname{sech} \eta\left(\theta-\theta_{0}\right) .
$$

Using the results given in Eqs.(32) and (36) we write down the final form of the first order perturbed soliton $U=\left(\hat{U}_{0}+\gamma\left(\phi_{1}+i \psi_{1}\right)\right) \exp \left[i \xi\left(\theta-\theta_{0}\right)+i\left(\sigma-\sigma_{0}\right)\right]$ (by choosing $\gamma=1$ ) as

$$
\begin{aligned}
U= & {\left[\eta \operatorname{sech} \eta\left(\theta-\theta_{0}\right)+c\left[1-\left(\theta-\theta_{0}\right) \tanh \eta\left(\theta-\theta_{0}\right)\right]\right.} \\
& \left.+i \frac{\eta}{2}\left(\theta-\theta_{0}\right)^{2} \operatorname{sech} \eta\left(\theta-\theta_{0}\right)\right] \exp \left[i \xi\left(\theta-\theta_{0}\right)+i\left(\sigma-\sigma_{0}\right)\right] .
\end{aligned}
$$

In Fig. 6, we have plotted the square of the absolute value of the perturbed soliton i.e. $|U|^{2}$ from Eq.(37). From the figure, we observe that the amplitude of the soliton decreases as time progresses, because of the damping due to viscosity of the surrounding medium. Therefore, when the viscosity is high the soliton is expected to travel only for a short time and will stop after that. On the other hand, when the viscosity is low, the soliton will travel for some time. Similar results have also been observed by Yakushevich [27] through numerical analysis. They showed that when the viscosity is low, the soliton passes more than 3000 chain links in DNA like a heavy Brownian particle and when the viscosity is large, the soliton stops after a few chain links. 




$\mathrm{Z}$

FIG. 4: Square of absolute value of the perturbed soliton solution (Eq. (37)) under viscous damping.

\section{CONCLUSION}

In this paper, we investigated the nonlinear dynamics of a protein-DNA molecular system under thermal fluctuations in a viscous surrounding medium by considering DNA as a set of two coupled linear chains and protein as a single linear molecular chain interacting through linear coupling. In the non-viscous limit, the dynamical equation for the system is derived from the Hamiltonian through a semiclassical approach using Glauber's coherent state method combined with Holstein-Primakoff (H-P) bosonic representation under continuum approximation. The equation of motion reduces to a set of coupled equations in which the equation for DNA dynamics is a nonlinear equation for rotation of bases and inhomogeneous wave equations representing vibration of hydrogen atom in the bases and for the protein molecule. In the linear limit, the above equations are decoupled and reduced to timedependent Schrödinger equation for a free particle and one-dimensional homogeneous linear wave equations. While the former one admits dispersive plane transverse wave solution the later ones give non-dispersive wave solutions. When protein molecule and thermal phonons started interacting with the DNA, the coupling introduces nonlinearity into the dynamics of bases in DNA, and the set of coupled equations of motion reduce to the completely integrable nonlinear Schrödinger equation that admits N-soliton solutions. During interaction the energy of the excited DNA molecule increases and the nonlinearity localizes the energy thus forming localized solitons. The solitons represent opening of base pairs in both the strands which collectively form a bubble travelling along the DNA double helical chain at physiolog- 
ical temperature. Thus, the protein molecule acts as a zip runner that opens the base pairs which close when the protein molecule progress along the DNA chain. For, strong coupling, the amplitude of the soliton is expected to decrease. On the other hand, the dynamics of the system in the viscous medium is governed by a perturbed nonlinear Schrödinger equation. The effect of viscosity is understood by carrying out a multiple scale perturbation analysis. The results show that while the amplitude of the soliton decreases and the velocity remains constant as time progresses. The soliton, damps out quickly in the case of high viscosity and moves for some time when the viscosity of the surrounding medium is low. The events that happen in the present study may represent the binding of an RNA-polymerase to a promoter site in the DNA during the transcription process. Our results have very strong coincidence with the experimental data $[52,53,54,55,56]$ that the binding of RNA-polymerase to the promoter site in DNA is accompanied by a local distorsion of the DNA bases in the form of solitons which can propagate along the DNA double helix. In nature, protein binds to DNA in a very specific site like promoter, coding or terminator which has a specific sequence of bases and this makes the strands site-dependent or inhomogeneous. Hence, it is important

to understand the nonlinear dynamics of inhomogeneous DNA with the protein bound to specific site of DNA and the study is under progress.

\section{Acknowledgements}

The work of M. D and V.V forms part of a major DST project.

[1] L. Stryer, Biochemistry. $4^{\text {th }}$ ed (W. H. Freeman and Company, New York, 1995).

[2] S. J. van Noort, K. O. van der Werf, A. P. M. Eker, C. Wyman, B. G. de Grooth, N. F. van Hulst, and J. Greve, Biophys. J. 74, 2840 (1998).

[3] S. J. Koch and M. D. Wang. Phys. Rev. Lett. 91, 028103 (2003).

[4] S. Klimasauskas, S. Kumar, R. J. Roberts and X. Cheng, Cell, 36, 357 (1994).

[5] S. Klimasauskas, T. Szyperski, S. Serva and K. Wuthrich, EMBO J. 17, 317 (1998).

[6] K. Liebert, A. Hermann, M. Schlickenrieder and A. Jeltsch, J. Mol. Biol. 341, 443 (2004).

[7] N. Shimamoto, J. Biol. Chem. 274, 15293 (1999).

[8] N. Huang, N. K. Banavali and A. D. MacKerell Jr, Proc. Natl. Acad. Sci. 100, 68 (2003). 
[9] J. R. Horton, G. Ratner, N. K. Banavali, N. Huang, Y. Choi, M. A. Maier, V. E. Marquez, A. D. MacKerell Jr and X. Cheng, Nucleic Acids Res. 32, 3877 (2004).

[10] N. Huang and A. D. MacKerell Jr, J. Mol. Biol. 345, 265 (2005).

[11] M. Karplus and G. A. Petsko, Nature, 347, 631 (1990).

[12] S. W. Englander, N. R. Kallenbanch, A. J. Heeger, J. A. Krumhansl and S. Litwin, Proc. Natl. Acad. Sci. U.S.A 77, 7222 (1980).

[13] S. Yomosa, Phys. Rev. A 27, 2120 (1983).

[14] S. Yomosa, Phys. Rev. A 30, 474 (1984).

[15] S. Takeno and S. Homma, Prog. Theor. Phys. 70, 308 (1983).

[16] S. Takeno and S. Homma, Prog. Theor. Phys. 72, 679 (1984).

[17] M. Peyrard and A. R. Bishop, Phys. Rev. Lett. 62, 2755 (1989).

[18] P. L. Christiansen, P. C. Lomdahl and V. Muto, Nonlinearity, 4, 477 (1991).

[19] L. V. Yakushevich, Nanobiology, 1, 343 (1992).

[20] J. A. Gonzalez and M. M. Landrove, Phys. Lett. A 292, 256 (2002).

[21] M. Peyrard, Nonlinearity, 17, R1 (2004).

[22] M. Daniel and V. Vasumathi, Physica D, 231, 10 (2007).

[23] M. Daniel and V. Vasumathi, Phys. Lett. A 372, 5144 (2008).

[24] A. Campa, Phys. Rev. E 63, 021901 (2001).

[25] S. Cocco, R. Monasson and J. F. Marko, Phys. Rev. E 65, 041907 (2002).

[26] S. Cocco , R. Monasson and J. F. Marko, Phys. Rev. E 66, 051914 (2002).

[27] L. V. Yakushevich, A. V. Savin and L. I. Manevitch, Phys. Rev . E 66, 016614 (2002).

[28] S. Zdravkovic and M. V. Sataric, Phys. Scr. 64, 612 (2001).

[29] S. Zdravkovic and M. V. Sataric, J. Mod. Phys. B 17, 5911 (2003).

[30] C. B. Tabi, A. Mohamadov and T. C. Kofane, J. Math. Biosci. Eng. 5, 205 (2008).

[31] A. S. Davydov, Ukr. Fiz. Zh. 20, 179 (1975).

[32] A. S. Davydov, Solitons in Molecular Systems (Reidel, Dordrecht, 1985).

[33] A. C. Scott, Physica Scripta 29, 279 (1984).

[34] J. X. Xiao and L. Yang, Phys. Rev. A 44, 8375 (1991).

[35] M. Daniel and K. Deepamala, Physica A 240, 526 (1997).

[36] M. Daniel and M. M. Latha, Physica A 298, 351 (2001).

[37] S. Yomosa, J. Phys. Soc. Jpn. 53, 3692 (1984). 
[38] M. V. Sataric, L. Matsson, and J. A. Tuszynski, Phys. Rev. E 74 , 051902 (2006).

[39] M. D. Betterton and F. Julicher, Phys. Rev. Lett. 91, 258103 (2003).

[40] J. Yan and J. F. Marko, Phys. Rev. E 68, 011905 (2003).

[41] S. M. Bhattcharjee, Europhys. Lett. 65, 574 (2004).

[42] F. Habib and R. Bundschuh, Phys. Rev. E 72, 031906 (2005).

[43] R. Murugan, Phys. Rev. E 76, 011901 (2007).

[44] M. V. Sataric and J. A. Tuszynski, Phys. Rev. E 65, 051901 (2002).

[45] M. Daniel and L. Kavitha, Phys. Lett. A 295, 121 (2002) and references therein.

[46] N. S. Fialko and V. D. Lakhno, Phys. Lett. A 278, 108 (2000).

[47] A. M. Guo and H. Xu, Phys. Lett. A 364, 48 (2007).

[48] T. Holstein and H. Primakoff, Phys. Rev. 58, 1098 (1940).

[49] R. J. Glauber, Phys. Rev. 131, 2766 (1963).

[50] V. E. Zakharov and A. B. Shabat, Sov. Phys. JETP 34, 62 (1972).

[51] T. Ha, I. Rasnik, W. Cheng, H.P. Babcock, G. H. Gauss, T. M. Lohman and S. Chu, Nature, 419, 638 (2002).

[52] U. Siebenlist, R. B. Simpson and W. Gilbert, Cell 20, 269 (1980).

[53] U. Siebenlist, Nature 279, 651 (1979).

[54] M. Sluyser, Trends Biochem. Sci. 8, 236 (1983).

[55] J.W. Saucier and J. C. Wang, Nature. New Biol. 239, 167 (1972).

[56] S. G. Kamzolova, Stud. Biophys. 87, 175 (1982).

[57] M. Peyrard, J. Biol. Phys. 27, 217 (2001).

[58] M. Daniel and J. Beula, Phys. Rev. B 77, 144416 (2008). 